\title{
In Silico Study of Vacuolin-1 as an Inhibitor of HSP27 for Precancerous Treatment of Breast Cancer
}

\author{
Diah Eka Agustin ${ }^{1}$, Mumtaz Nabila Ulfah ${ }^{1}$, Siti Nur Aisyah ${ }^{1}$, Pamuji Lestari Arumsari ${ }^{1}$, Kadita \\ Octavia Pertiwi ${ }^{1}$, Fatchiyah Fatchiyah ${ }^{1,2 *}$ \\ ${ }^{1}$ Department of Biology, Faculty of Mathematics and Natural Sciences, Brawijaya University, Jalan Veteran, Malang, East Java, \\ Indonesia, 65145 \\ ${ }^{2}$ Research center of SMONAGENES, Brawijaya University, Jalan Veteran, Malang, East Java, Indonesia, 65145
}

Submission: 07 July 2021; Revised: 16 July 2021; Accepted: 30 July 2021

*Corresponding author: Fatchiyah Fatchiyah; e-mail: fatchiya@ub.ac.id; tel.: 082142583774

\begin{abstract}
Breast cancer has a great chance of being cured if it is diagnosed and treated properly in its early stage. The pre-cancer stage is an early stage of cancer development characterized by the overexpression of HSP27. Therefore, HSP27 can be a therapeutic target of cancer. This study aims to analyze whether vacuolin-1, a small drug compound known for its ability to inhibit metastasis, can inhibit HSP27 to prevent precancerous development in breast cancer, as well as its ADME and biosafety aspects. Protein \& ligand structures were obtained from RCSB PDB and PubChem database. Preparation was performed with Discovery Studio and PyRx. Drug-likeness/ADME analysis was performed in Swiss-ADME web server. Biosafety analysis was performed in MetaTox web server. Molecular docking was performed using PyRx. The visualization of docking results was performed using Discovery Studio. The docking result between vacuolin-1 and HSP27 showed that vacuolin-1 can act as an HSP27 inhibitor by interacting with S78 residue of HSP27 and blocking its phosphorylation as well as depolymerization process. The drug-likeness characterization result of this compound showed that vacuolin-1 violates one of the four Lipinski's Rule of Five. Biosafety analysis showed that vacuolin-1 has a low toxicity level with an estimated LD50 around 13,016.65 mg/kg.
\end{abstract}

Keywords: breast cancer, HSP27, molecular docking, pre-cancer, vacuolin-1

\section{INTRODUCTION}

Cancer is the leading cause of death in the world, and the number of cancer deaths in 2020 is close to 10 million. Breast cancer is a form of cancer with high morbidity and death rates. There is a high possibility of a cure if cancer is detected and treated early on [1]. The pre-cancer stage is an early stage of cancer development characterized by overexpression of HSP27. Overexpression of HSP27 in cancer cells is associated with a poor prognosis because it protects malignant cells that cause cancer so that they cannot carry out the apoptosis process. It was also associated with drug resistance and radioresistance, resulting in poor response when patients received various treatments, including chemotherapy and radiotherapy. It is also associated with aggressive cell growth and infiltration with poor prognosis [2]. HSP27 might be a target for cancer cell treatment [3].

HSP27 itself is an sHSP (small heat shock protein) found in mammals. HSP27 in the human body is encoded by the HSPB 1 gene and includes a single-copy gene with a $2.2 \mathrm{~kb}$ transcript, which is organized into three exons in a 205 amino acid protein pair. HSP27 is mainly located in the cytoplasm, but it can be located in the nucleus due to exposure to stress conditions. The main function of HSP27 is to regulate protein folding, immune response, promote cancer cells, induce anti-cancer drug resistance, aging, biomarkers of various diseases, neurodegenerative diseases, cell development, and differentiation through molecular chaperone activity. HSP27 overexpression can be related to tumor occurrence and metastasis so its overexpression can lead to cancer development [4].

Vacuolin-1 is a small molecule drug that can significantly inhibit metastasis. The compound showed significant ability to inhibit the formation, migration, and invasion of various types of cancer cell colonies in vitro. Vacuolin-1 strongly inhibits lysosomal fusion of the autophagosome and lysosomal degradation of the endosome. The compound also affects focal adhesion (FA) assembly and disassembly kinetics by inhibiting the recovery and degradation of integrins. It also substantially reduced breast cancer or melanoma metastasis and/or tumor development in numerous studies or transgenic animal models [5]. This study aimed to analyze whether vacuolin-1 can inhibit HSP27 to prevent precancerous development in breast cancer as well as its druglikeness and biosafety aspects.

\section{RESEARCH METHODS}

\section{Receptor and ligand preparation}

The structure of HSP27 (PDB ID: 6DV5)

was downloaded from the PDB database 
(www.rcsb.org), then the preparation was performed with Biovia Discovery Studio Visualizer $\mathrm{v} 21.1 .0$ to remove its ligand contaminants and water molecules. The structure of the small drug molecule vacuolin-1 (CID: 9661141) was downloaded from the PubChem database as 3D-SDF, then its energy was minimized and converted to PDB format using PyRx [6].

\section{ADME/drug-likeness}

Drug-likeness analysis, which was performed to see if vacuolin-1 meets Lipinski's Rule of Five (Ro5), was carried out in the SwissADME web server (http://www.swissadme.ch/) [7].

\section{Biosafety analysis}

The biosafety analysis of Vacuolin-1 was performed in the MetaTox web server (http://way2drug.com/mg) [8].

\section{Molecular docking \& visualization}

Protein-ligand specific docking was performed using Pyrx with grid center $X$ : 164.9846, Y: 393.7237, Z:-114.4921 (HSP27Vacuolin-1) and X:-164.87, Y: 394.572, Z:114.6670 (HSP27-Ivermectin/control). The docking results (ligand only) are then combined with protein (macromolecules) using PyMOL software [9]. Visualization was performed with Biovia Discovery Studio Visualizer v 21.1.0 [10].

\section{RESULTS AND DISCUSSIONS}

The docking result between vacuolin-1 and Hsp27 showed that both molecules can bind to each other (Figure 1). The binding energy involved in this interaction was $-3.82 \mathrm{kcal} / \mathrm{mol}$. This interaction revealed 3 amino acids residues that bind to Hsp27. These amino acid residues are Ser78, Arg79, and Ser83. Several categories of interactions occur between vacuolin-1 and these amino acid residues including hydrophobic interaction and hydrogen bond (Table 1). These non-bond/non-covalent interactions can promote ligand binding affinity with the protein and stabilize it in certain conditions [11]. For instance, researchers have demonstrated that, hydrogen bonds may strengthen the receptor-ligand interaction formed if both electron acceptor and donor atoms have hydrogen-bonding abilities that are significantly stronger or weaker than the hydrogen and oxygen atoms present in water. On the contrary, if the hydrogen bonds present are a mixture of strong-weak H-bond pairs, interference by bulk water can occur, causing the ligand bond affinity to decrease. [12].

Vacuolin-1 can interact and bind to the serine 78 residue in the HSP27 phosphorylation pocket. Previous studies have reported that there are three serine residues (Ser15, Ser78, and Ser82) and a threonine residue (Thr143) through which multiple kinases can phosphorylate this protein. Moreover, Ser78 and Ser82 have been reported to significantly contribute to the oligomerization of HSP27. HSP27 oligomerization is reversibly regulated by its phosphorylation and dephosphorylation events. HSP27 phosphorylation stimulates the formation of small oligomers, while its dephosphorylation stimulates the formation of large oligomers.[4].

In this study, Ivermectin, one of the antiparasitic drugs approved by the Food and Drug Administration (FDA), known for its ability to inhibit oncogenic pathway signaling through HSP27 [13], was used as a control. Ivermectin can bind to the phosphorylation pocket of HSP27 at the same residue as vacuolin-1. This interaction inhibits the phosphorylation and depolymerization of HSP27. Molecular binding to the phosphorylation pocket of HSP27 can block HSP27-regulated survival signaling and clientoncoprotein interactions. Therefore, the binding of vacuolin-1 to this site can inhibit pre-cancerous development in breast cancer cases.

HSP27 can be found in many cell types, including adipose cells which are found in the breast. HSP27 is mainly located in the cytoplasm and can be found in the perinuclear region, endoplasmic reticulum, and nucleus $[14,15,16]$. Cytoplasmic HSP27 can be found in interphase cells. In mitotic cells, it is co-localized with mitotic spindles. During heat shock stimulation, it's translocated to the nucleus then resided in subnuclear structures known as SC35 speckles (nuclear splicing speckles). As previously explained, vacuolin-1 as an inhibitor of HSP27 can interact with some residues located in the phosphorylation pocket of HSP27. This direct binding, as seen in ivermectin as well, may inhibit MAPKAPK2-mediated HSP27 phosphorylation and depolymerization [17]. This mechanism occurs in the cytoplasm so vacuolin-1 doesn't need any intermediary protein or agent.

Vacuolin-1 was analyzed based on the SwissADME database regarding several physicochemical properties including molecular weight (MW), LogP value, donor hydrogen bonds number, acceptor hydrogen bonds number, rotatable bonds number, and total polar surface area (TPSA) (Table 2). 
Table 1. Interaction of ivermectin-HSP27 (control) and vacuolin-1-HSP27

\begin{tabular}{|c|c|c|c|c|c|}
\hline $\begin{array}{l}\text { Ligand- } \\
\text { Protein } \\
\text { Complex }\end{array}$ & $\begin{array}{c}\text { Binding } \\
\text { Energy } \\
\text { (kcal/mol) }\end{array}$ & Point Interaction & Distance & Category & Types \\
\hline \multirow{12}{*}{$\begin{array}{l}\text { Ivermectin } \\
\text { Hsp27 } \\
\text { (control) }\end{array}$} & \multirow[t]{12}{*}{0,97} & C:SER78:OG - :LIG2:O & 2,95299 & Hydrogen Bond & $\begin{array}{l}\text { Conventional Hydrogen } \\
\text { Bond }\end{array}$ \\
\hline & & D:ARG56:NE - :LIG2:O & 3,1767 & Hydrogen Bond & $\begin{array}{l}\text { Conventional Hydrogen } \\
\text { Bond }\end{array}$ \\
\hline & & :LIG2:HO - :LIG2HO & 1,78226 & Hydrogen Bond & $\begin{array}{l}\text { Conventional Hydrogen } \\
\text { Bond }\end{array}$ \\
\hline & & :LIG2:C - C:SER82:O & 3,17478 & Hydrogen Bond & Carbon Hydrogen Bond \\
\hline & & :LIG2:C - C:LEU77:O & 3,24069 & Hydrogen Bond & Carbon Hydrogen Bond \\
\hline & & :LIG2:C - :LIG2:O & 3,7589 & Hydrogen Bond & Carbon Hydrogen Bond \\
\hline & & C:LEU77 - :LIG2 & 5,4245 & Hydrophobic & Alkyl \\
\hline & & :LIG2:C - C:LEU77 & 4,56104 & Hydrophobic & Alkyl \\
\hline & & :LIG2:C - D:ARG56 & 4,30842 & Hydrophobic & Alkyl \\
\hline & & :LIG2:C - C:LEU10 & 4,94325 & Hydrophobic & Alkyl \\
\hline & & :LIG2:C - C:LEU77 & 4,26871 & Hydrophobic & Alkyl \\
\hline & & D:TYR54 - :LIG2:C & 4,9115 & Hydrophobic & Pi-Alkyl \\
\hline \multirow[t]{7}{*}{$\begin{array}{l}\text { Vacuolin-1- } \\
\text { Hsp27 }\end{array}$} & \multirow[t]{7}{*}{3,82} & :LIG1:H - C:SER78:O & 1,86251 & Hydrogen Bond & $\begin{array}{l}\text { Conventional Hydrogen } \\
\text { Bond }\end{array}$ \\
\hline & & :LIG1:H - C:ARG79:O & 2,05026 & Hydrogen Bond & $\begin{array}{l}\text { Conventional Hydrogen } \\
\text { Bond }\end{array}$ \\
\hline & & :LIG1:HN - C:SER78:O & 2,43165 & Hydrogen Bond & $\begin{array}{l}\text { Conventional Hydrogen } \\
\text { Bond }\end{array}$ \\
\hline & & :LIG1:HN-C:ARG79:O & 2,66486 & Hydrogen Bond & $\begin{array}{l}\text { Conventional Hydrogen } \\
\text { Bond }\end{array}$ \\
\hline & & C:SER83:CB - :LIG1:O & 3,15669 & Hydrogen Bond & Carbon Hydrogen Bond \\
\hline & & $\begin{array}{l}\text { C:SER78:C,O;ARG79:N } \\
\text {-:LIG1 }\end{array}$ & 3,94123 & Hydrophobic & Amide-Pi Stacked \\
\hline & & :LIG1 - C:ARG79 & 3,82539 & Hydrophobic & Pi-Alkyl \\
\hline
\end{tabular}

The drug-likeness characterization result showed that vacuolin-1 had violated one of Lipinski's Five Rules. The violation of this rule is because the molecular weight of vacuolin-1 is higher than the rule of Ro5 (the molecular weight of vacuolin-1 is $577.42 \mathrm{~g} / \mathrm{mol}$, more than the required number of $500 \mathrm{~g} / \mathrm{mol}$ ), but according to Veber's provisions, vacuolin-1 is appropriate with criteria. All compounds which have the same rules as Lipinski and Veber's rules show that these compounds can theoretically be said to have ideal oral bioavailability to be used as new drugs [17]. The drug-likeness of a compound that has the potential to become a drug can be determined using the Ro5 (Rules of five) by Lipinski and his colleagues which can show that the molecule of the drug can be distinguished based on the ability to absorb orally or not. According to Ro5, if a molecule violates two or more of these four rules, it will not have oral activity [18]. Ro5 predicts that when the donor is greater than $5 \mathrm{H}$, the molecular weight is greater than $500 \mathrm{~g} / \mathrm{mol}$, the calculated $\log \mathrm{P}$ is 
greater than 5, and the hydrogen bond acceptor is greater than 10 , the oral drug absorption or compound penetration is poor or unsatisfactory [19].

\section{Vacuolin-1 + HSP27}

A

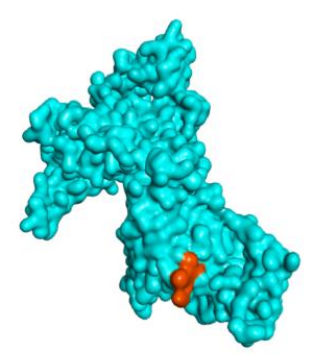

\section{HSP27 + Ivermectin}

A

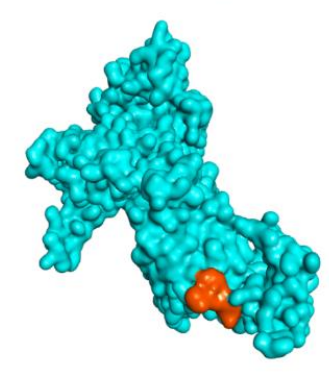

B

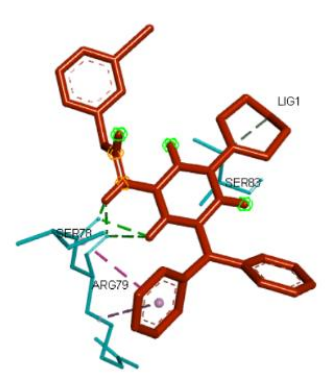

B

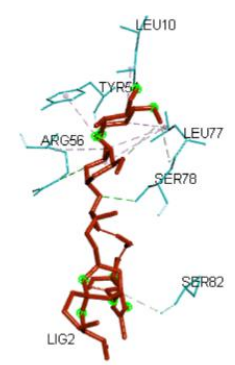

C

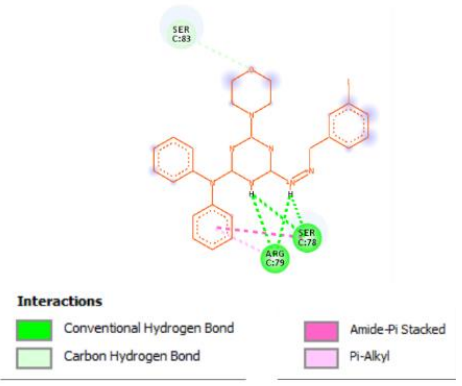

C

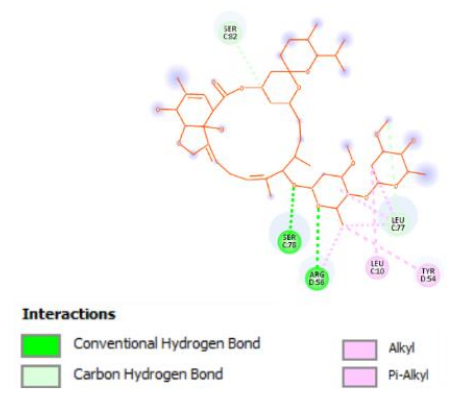

Figure 1. Docking result. (1) interaction of HSP27 and vacuolin-1, (2) interaction of HSP27 and Ivermectin. (A) binding location, (B) ligand interaction with amino acid residues of HSP27, (C) 2D diagram of the interaction.

Based on these provisions, vacuolin-1 can be used as a new drug candidate but its oral absorption may not be ideal because it violates one of the Ro5 provisions. The results of drug similarity characterization showed that vacuolin-1 had violated one of Lipinski's Five Rules. The violation of this rule is because the molecular weight of vacuolin-1 is higher than the rule of Ro5 (the molecular weight of vacuolin-1 is 577.42 $\mathrm{g} / \mathrm{mol}$, more than the required number of 500 $\mathrm{g} / \mathrm{mol}$ ), but according to Veber's provisions, vacuolin-1 is appropriate with criteria. All compounds that have the same rules as Lipinski and Veber's rules show that these compounds can theoretically be said to have ideal oral bioavailability to be used as new drugs [17]. Further research on Vacuolin-1, using both in vitro and in vivo methods can be developed in such a way that the oral bioavailability of Vacuolin-1 can be even better. MetaTox is a software-based program that generates metabolites and calculates the probability of their formation when a generation of metabolic pathways is built into acute toxicity prediction. The metabolic predictions included in MetaTox use biotransformation dictionaries based on preliminary predictions of possible types of biotransformation, which also describe the metabolic profiles of the compounds. SMILE mycotoxin standard used to predict metabolic products in MetaTox [8].

Table 2. Drug likeness of Vacuolin-1 based on SwissADME

\begin{tabular}{l|c}
\hline Compound & Vacuolin-1 \\
\hline CID & 9661141 \\
\hline MW (g/mol) & 577.42 \\
\hline n Rotatable Bond & 7 \\
\hline n H-bond Acceptor & 5 \\
\hline n H-bond Donor & 1 \\
\hline Log P & 4.54 \\
\hline TPSA $\left(\AA^{\mathbf{2}}\right)$ & 78.77 \\
\hline
\end{tabular}

Table 3. Toxicity prediction of vacuolin-1 based on MetaTox

\begin{tabular}{l|c}
\hline Compound & Vacuolin-1 \\
\hline $\begin{array}{l}\text { Predicted } \\
\text { LD50 (mg/kg) }\end{array}$ & $13,016.65$ \\
\hline Category & Low toxic \\
\hline Adverse Effect (Pa score) & 0.420 \\
\hline $\begin{array}{l}\text { Adverse Effect (side } \\
\text { effect) }\end{array}$ & Hepatotoxicity \\
\hline Mutagenicity (Pa score) & - \\
\hline Mutagenicity (side effect) & - \\
\hline
\end{tabular}


Vacuolin-1 belongs to the low toxic category with a predicted LD50 of 13,016.65 $\mathrm{mg} / \mathrm{kg}$. Because vacuolin-1 is categorized as low toxic, it is almost impossible that the compound will be toxic to the human body [20]. The adverse effect of the vacuolin-1 compound is hepatotoxicity with a $\mathrm{Pa}$ score of 0.420 . This compound cannot act as a mutagenic agent (Table 3). Prediction metabolites for vacuolin-1 come from reactions of $\mathrm{N}$-Hydroxylation (1st generation to 1), N-Oxidation (1st generation to 1), NGlucuronidation (1st generation to 2), and NDealkylation (1st generation to 3-14).

\section{CONCLUSION}

The small drug molecule vacuolin-1 compound is a potent inhibitor of HSP27 because it can interact and bind to the serine 78 residue in the HSP27 phosphorylation pocket. The toxicity of the vacuolin-1 is in the low toxic category with an estimated LD50 of $13,016.65 \mathrm{mg} / \mathrm{kg}$. The detrimental effect of the vacuolin-1 compound is hepatotoxicity with a Pa score of 0.420 . However, the drug-similarity characterization of vacuolin-1 shows that this compound violates one of four Lipinski's Rule of Five which is because of its higher molecular weight than the Ro5 rule.

\section{ACKNOWLEDGEMENT}

The authors would like to thank all Bioinformatics lecturers and laboratory practice assistants for their contribution in terms of guidance, direction, support, and suggestions for this research.

\section{REFERENCES}

[1]. WHO. Cancer. 2021. www.who.int/newsroom/fact-sheets/detail/cancer accessed on 20 April 2021.

[2]. Kim,E., Lee,H., Lee, D.H. Sangwoo B., JaeWon, S., Dooil, J., Joon, K., Chul-Koo, C., Yoon-Jin, L and Yun-Sil, L. 2007. Inhibition of Heat Shock Protein 27-Mediated Resistance to DNA Damaging Agents by a Novel PKC $\delta$-V5 Heptapeptide. Cancer Res. 67(13):6333-6341.

[3]. Vidyasagar, A. Wilson, N.A. \& Djamali, A. Heat shock protein 27 (HSP27): biomarker of disease and therapeutic target. Fibrogenesis \& Tissue Repair. 5(7): 1-7.

[4]. Choi, S. K., Kam, H., Kim, K. Y., Park, S. I., \& Lee, Y. S. 2019. Targeting Heat Shock Protein 27 in Cancer: A Druggable Target for Cancer Treatment. Cancers. 11(8): 1195. https://doi.org/10.3390/cancers11081195.
[5]. Ye, Z., D. Wang, Y. Lu, Y. He, J. Yu, W. Wei, C. Chen, R. Wang, L. Zhang, L. Zhang, M. T. N. Le, W. C. Cho, M. Yang, H. Zhang, \& J. Yue. 2021. Vacuolin-1 inhibits endosomal trafficking and metastasis via CapZß. Oncogene. 40: 1775-1791.

[6]. Dallakyan S., \& Olson A. J. 2015. SmallMolecule Library Screening by Docking with PyRx. Methods Mol. Biol. 1263:243- 250.

[7]. Chagas, C.M, Moss. S \& Alisaraie, L. 2018. Drug metabolites and their effects on the development of adverse reactions: Revisiting Lipinski's Rule of Five. Int $J$ Pharm.1(2):133-149.

[8]. Agahi, Fojan., Cristina Juan., Guillermina Font., Ana Juan-García., 2020. In silico methods for metabolomic and toxicity prediction of zearalenone, $\alpha$-zearalenone, and $\beta$-zearalenone, Food and Chemical Toxicology, 146.

[9]. Yuan, S., Chan, H. \& Hu, Zhenquan. 2017. Using PyMOL as a platform for computational drug design. Wiley Interdisciplinary Reviews: Computational Molecular Science. $7 . \quad$ e1298. 10.1002/wcms.1298.

[10]. Dassault Systemes BIOVIA. 2017. BIOVIA Discovery Studio 2016 [Computer Software]. Retrieved from https://3dsbiovia.com.

[11]. Bare, Y., L. I. N. Indahsari, D. R. T. Sari, \& T. Watuguly. 2021. In silico study: potential prediction of Curcuma longa and Cymbopogon citratus essential oil as lipoxygenase inhibitor. Journal of Smart Bioprospecting and Technology. 2(2): 75-80.

[12]. Chen, D., N. Oezguen, P. Urvil, C. Ferguson, S. M. Dann, \& T. C. Savidge. 2016. Regulation of protein-ligand binding affinity by hydrogen bond pairing. Science Advances. 2 (3): e1501240. doi: 10.1126/sciadv. 1501240.

[13]. Nappi, L., A. H. Aguda, N. Al Nakouzi, B. Lelj-Garolla, E. Beraldi, N. Lallous, M. Thi, S. Moore, L. Fazli, D. Battsogt, S. Stief, F. Ban, N. T. Nguyen, N. Saxena, E. Dueva, F. Zhang, T. Yamazaki, A. Zoubeidi, A. Cherkasov, G. D. Brayer, \& M. Gleave. 2020. Ivermectin inhibits HSP27 and potentiates efficacy of oncogene targeting in tumor models. The Journal of Clinical Investigation. 130(2):700-714. https://doi.org/10.1172/JCI130819.

[14]. Hino, M., Kurogi K., Okubo M.-A., MurataHori M., \& Hosoya H. 2000. Small heat shock protein 27 (HSP27) associates with tubulin/microtubules in HeLa cells. Biochem. Biophys. Res. Commun. 271:164-169.

[15]. Echaniz-Laguna, A., Geuens T., Petiot P., Pereon Y., Adriaenssens E., Haidar M., Capponi S., Maisonobe T., Fournier E., 
Dubourg O., Degos B., Salachas F., Lenglet T., Eymard B., Delmont E., Pouget J., Juntas Morales R., Goizet C. \& Stojkovic T. 2017. Axonal Neuropathies due to Mutations in Small Heat Shock Proteins: Clinical, Genetic, and Functional Insights into Novel Mutations. Hum. Mutat. 38:556-568.

[16]. Vos, M.J., Kanon B., \& Kampinga H.H. 2009. HSPB7 is a SC35 speckle resident small heat shock protein. Biochim. Biophys. Acta. 1793:1343-1353.

[17]. Zerroug, A. Samir, Chtita, Belaidi, S. Benbrahim, I. \& Sinha, L. 2019. Virtual screening in drug-likeness and structure/activity relationship of pyridazine derivatives as Anti-Alzheimer drugs. Science. 31(4): 595-601
[18]. Guan, L. Yang, H. Cai, Y. Sun, L. Di, P. Li, W. Liu, G. \& Tang, Y. 2019. ADMET-scorea comprehensive scoring function for evaluation of chemical drug-likeness. Medchemcomm. 148-157.

[19]. Benet, L. Z., Hosey, C.M., Ursu, O., \& Oprea, T.I.. 2016. BDDCS, the rule of five and drug ability. Advanced Drug Delivery Reviews. 1(101): 89-98.

[20]. Lu, Yingying., Shichen D., Baixia H., Chang L., Kaiyuan Zhu., Wenjing G., Qian W., King-Ho C., Connie WM W., Wu-Tian W., Huss M., Jianbo Y., 2014. Vacuolin-1 potently and reversibly inhibits autophagosome-lysosome fusion by activating RAB5A. Autophagy, 10(11):18951905. 